\title{
Analysis of IFMBE-CED 2017 Worldwide Clinical Engineering Survey
}

\author{
By L. Nascimento' ${ }^{1}$, S. Calil' ${ }^{1}$, T. Judd ${ }^{2}$, Y. David ${ }^{3}$ \\ ${ }^{1}$ Department of Biomedical Engineering - School of Electrical and Computer Engineering. University of Campinas, Brazil \\ ${ }^{2}$ Chair, IFMBE Clinical Engineering Division. Associate Editor, Health Technology and Quality, The Permanente Journal \\ ${ }^{3}$ Biomedical Engineering Consultants, LLC, University of Texas School of Public Health
}

\begin{abstract}
Background and Objective: Clinical engineering (CE) professionals are fundamental to the deployment of healthcare technology and the management of its life cycle. As the role of technology grows in healthcare, so does the need for trained $\mathrm{CE}$ practitioners and the dynamic nature of the domain requires them to maintain their skills. However, the skills and activities required from clinical engineers around the world are not homogeneous, so the CE Division at IFMBE promoted a global survey to identify a common body of knowledge and body of practices for the profession.

Material and Methods: This survey, based on a previous one conducted by the American College of Clinical Engineering, was aimed at collecting data about clinical engineering practices and the importance of certain competencies for their practitioners.

Results: Survey results indicate the profession still maintains certain traditional characteristics, such as the predominance of professionals with a background in electrical, electronic, or mechanical engineering and the prevalence of hospitals and clinics as employers. Some patterns in the perceived relevance of certain kinds of knowledge among different regions were also identified.

Conclusion: Overall, the survey seems adequate to reveal which skills and activities CEs considered the most relevant, but more responses are required before a solid Body of Knowledge and Body of Practice can be defined.

\section{Keywords - Clinical Engineering, Body of Knowledge, Body of Practice, Clinical Engineering Survey.}

Copyright (c) 2021. This is an open-access article distributed under the terms of the Creative Commons Attribution License (CC BY): Creative Commons - Attribution 4.0 International - CC BY 4.0. The use, distribution or reproduction in other forums is permitted, provided the original author(s) and the copyright owner(s) are credited and that the original publication in this journal is cited, in accordance with accepted academic practice. No use, distribution or reproduction is permitted which does not comply with these terms.
\end{abstract}

\section{INTRODUCTION}

To reap the full benefits of deploying technology in healthcare delivery, healthcare programs require competent clinical engineering professionals to manage its life cycle. As the technology's role rises over time so does the need for trained clinical engineering practitioners. Clinical Engineering (CE) is today one of the most dynamic professions in the world. ${ }^{1}$ This dynamic state challenges CE professionals to maintain their skills and stay current with the continuous progress of healthcare technologies. From the early stage of innovation throughout the rest of the technology life cycle, clinical engineers not only have to keep a constant update of their knowledge and expertise needed to develop their activities but also to periodically add, adapt, and learn new competencies and methodologies due to introduction of new and innovative technologies. Clinical engineers must sustain and further 
build their contribution to safe, efficient, and optimal life cycle stages and patient outcomes. ${ }^{2}$

While Maintenance Management, Equipment Specification, and Acquisition composed the basic set of knowledge for clinical engineers in the seventies and eighties, the current basic set is composed of more than 18 subjects and it is still growing. Table 1 compares the increasing number of subjects during the last 18 years.

TABLE 1. New Subjects Added to the Set of Knowledge of clinical engineering in the Last 18 Years (based on personal observations)

\begin{tabular}{|c|c|}
\hline $1970-1980$ & $1990-2015$ \\
\hline $\begin{array}{l}\text { - Medical Equipment } \\
\text { - } \text { Sanagement } \\
\text { - } \text { Procurement } \\
\text { - Education } \\
\text { - Individual Product } \\
\text { - } \text { Management } \\
\text { Individual Thinking }\end{array}$ & $\begin{array}{l}\text { - } \text { Medical Equipment Management } \rightarrow \\
\text { - Safety } \rightarrow \text { Risk Management } \\
\text { - Procurement } \\
\text { - } \text { Education } \\
\text { - } \text { Disaster Preparedness } \\
\text { - Cost Control (TCO. LCC) } \\
\text { - Technology Assessment } \\
\text { - Telemedicine (Homecare) } \\
\text { - Project Management } \\
\text { - Contract Management } \\
\text { - Mobile Healthcare (Events. } \\
\text { - Transports. Group Assistance) } \\
\text { - Home Care } \\
\text { - } \text { Information Technology } \\
\text { - Interoperability) } \\
\text { - Human Factor Engineering } \\
\text { - } \text { Forensic Analysis } \\
\text { - Systificial Intelligence Integration And Management } \\
\text { - Soft skills (Writing. Communication. } \\
\text { - Supervision) } \\
\text { Team Practicing }\end{array}$ \\
\hline
\end{tabular}

LCC = life cycle costs; TCO = total cost of ownership

There are very few recent studies describing worldwide activities developed by CEs. Most surveys were either done a long time ago ${ }^{3,4}$ or are recent, but regional. To our knowledge, there are no recent publications on surveys conducted to investigate $\mathrm{CE}$ activities worldwide. In 2004, a comprehensive CE survey ${ }^{5}$ was developed to address two points via two questionnaires. The first one investigated the structure, personnel, responsibilities, and resources of the CE departments all over the world. The second questionnaire was to investigate trends and current CE practices. To identify such practices, respondents were requested to check a list of several activities such as equipment management, quality control, risk management, education, and training. The resulting analysis from the 174 valid answers received, indicated that the main problems were lack of highly qualified personnel (because of the lack of quality academic programs), limited funding for technical training to maintain staff competencies for all equipment types and continuous pressure to reduce costs by increasing department efficiency. Additionally, the authors also concluded that despite the efforts for activities harmonization among CEs regarding the management of healthcare technology in hospitals all over the world, this subject remains non-uniform, with great variations in terms of structure, personnel, responsibilities, resources, and outcomes.

Starting in 2004, the BIOMEDEA project (a Europeanwide initiative) promoted the organization of three meetings aiming at the development and establishment of consensus on European guidelines and protocols for the harmonization and accreditation of high quality Medical and Biological Engineering and Science programs and for the training, certification and continuing education of professionals working in the health care systems.5 The third meeting took place through an international symposium on an important issue of quality assurance in biomedical/CE: patient safety.

In 2005, a meeting co-sponsored by the University of Stuttgart and the International Federation for Medical and Biological Engineering - IFMBE, ${ }^{6}$ produced several documents, which included an "Agreement for Mutual Recognition of Qualifications for Clinical Engineers". A white paper produced by the Clinical Engineering Division - CED/IFMBE7 described its contents. A further document was also produced: the "Protocol for the Training of Clinical Engineers in Europe."

Both were very important and valuable documents; however, some obstacles stopped the progress of such initiatives: 
1. There were no further discussions to carry on the work and the documents became just a source of consultation.

2. The documents were heavily based on the CE model adopted by the American College of Clinical Engineering (ACCE), meaning that it was an American model to be adopted in Europe.

3. The document aimed the CE in Europe and even though its development lacked further studies to understand the kind of CE activities practiced in many of the European countries.

4. The document contents would serve only as a source of consultation for countries outside Europe and it did not fill the need to find a worldwide harmonization for knowledge among CEs.

Then, also in 2005, CED/IFMBE launched a survey aiming to learn about the CE activities in different countries. ${ }^{8,9}$ The objective was to identify and develop a worldwide network of CEs and understand their activities. This survey looked for characteristics such as age, time of experience in the CE area, type of employer, primary position and all the activities developed within the work.

The results of this survey indicate some similarities among activities in several parts of the world. Figure 1 shows the results of the 2005 survey and it indicated that Technology Management was practiced by a range of 55\% (Asian CEs) to 85\% (North Americans and Canadian CEs) of the respondents. As another example, risk management practices varied from 39\% (Latin Americans CEs) to 70\% (North Americans and Canadians CEs). Such similarities can be the basis for developing stronger international cooperation among clinical engineers and CE professional organizations. This set of activities can also be the basis to understand the core of CE practices worldwide and develop a core of knowledge to be taught by any academic unit that aims to train CEs. It can also be used by countries/ societies that already have or are planning to develop a CE credentialing or certification system.

This was, however, a primary set of data. To have a more reliable set of the knowledge needed by CEs to develop not only their daily activities but to empower them to propose and develop advanced projects within the CE area, it was necessary to have a better understanding of the CE profile and practices worldwide. The results of the 2005 survey are outlined in Figure 1.

FIGURE 1. The results of the 2005 survey showing that, according to the respondents, Technology Management is one of the activities practiced by a range of 55\% (Asian CEs) to $85 \%$ (North American and Canadian CEs).

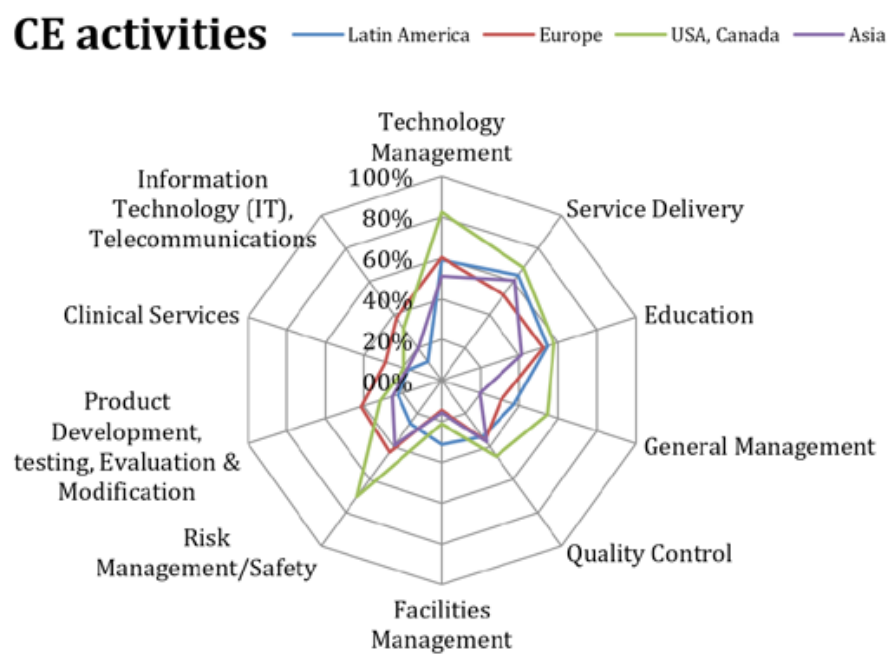

Ten years later, in 2017, sponsored by IFMBE/CED it was possible to develop and launch a worldwide project called "Body of Practice and Body of Knowledge - BoK \& BoP." This project, led by senior CEs from around the world, developed data collection tool (a survey), included additional questions designed not only to identify the CE activities practiced at their place of work but also what set of competencies is important for better development and successful outcomes from such activities.

This survey was based on a similar tool used by the ACCE to identify the profile and practices of CEs working in USA and Canada. ${ }^{10}$

\section{METHODS}

The topics and format of the questions were either extracted or modified from a survey kindly provided by the ACCE and Eng. Frank Painter. The original survey was used by ACCE to determine the current knowledge and skills needed for competent CE practice mainly in the United States and Canada. As the ACCE survey, this one was divided into five sections, aiming at collecting different pertinent types of information. 
The first part of the survey - "Contact Information", asked for identification and general data about the respondent. such as name, company, country and email address. In this part, only the country's name of the respondent was mandatory.

The second part - "Job Information," was focused on acquiring data about the CE employer and educational background. It included questions about the type of employer, about how respondents describe their profession, the primary nature of their current position, their academic background (engineering or other), and about the existence of CE certification in the country.

The third section - "Knowledge", presented a list of 28 knowledge topics and asked the respondents to rate the importance of those topics (Minor, Moderate, or High Importance) in the development of their activities.

The fourth part - "Responsibilities," presented eight classes of activities (Technology Management, Service Delivery Management, Product Development Management, IT/Telecommunications, Education, Facilities Management, Risk Management/Safety, and General Management). A list of multiple skills related to each one of these classes was then presented. and respondents were asked to rate how important (No, Minor, Moderate, or High importance) each skill is to develop each of the "Responsibility."

The final section of the questionnaire - "Work Activities", asked the respondents to indicate the percentage of time they dedicate to each one of the eight classes of activities presented in the previous part.

During the data analysis process, weights were assigned to the levels of importance indicated in the responses: for the knowledge topics where the answers had three rating levels; 0 (zero) was assigned for "Minor," 1 for "Moderate," and 2 for "High" importance.

For the responsibility topics. where four rating levels were presented. 0 (zero) was assigned for "No," 1 for "Minor," 2 for "Moderate," and 3 for "High."

Though there are several ways to present the data in this article, it was decided to show the result by geographical region: Latin America, Oceania, Asia, Middle East, Europe, Africa and, USA and Canada.
The survey was developed and presented to the invited participants with Google Forms and 574 invitations were sent by email.

\section{RESULTS}

From the 574 invitations to respond to the survey, 199 responses were received from 35 countries. From those; 35\% came from Latin America, 20\% from Oceania, 14\% from Asia, 11\% from the Middle East, 10\% from Europe, $6 \%$ from Africa and $4 \%$ from USA and Canada. Though it was below the expected number of responses, the results can already present important information regarding the objectives of the BoK \& BoP project. Due to the very low number of responses from USA and Canada (7 responses), here it will be left out of the resulting graphics but will be at a later point compared with the 2015 ACCE survey. ${ }^{8}$ The African Region also has few responses (12 responses) but is presented due to the very small number of CEs working in that region. The total number of respondents with an electrical/electronic/mechanical engineering (B.Sc.) degree is around $65 \%$.

As identified in the 2005 survey, the vast majority of CEs (48\%) are employed by hospitals or health clinics. Government agencies are ranked in the second position as employers according to $12.5 \%$ of the respondents (Figure 2).

FIGURE 2. Percentage of the types of employers of clinical engineers worldwide.

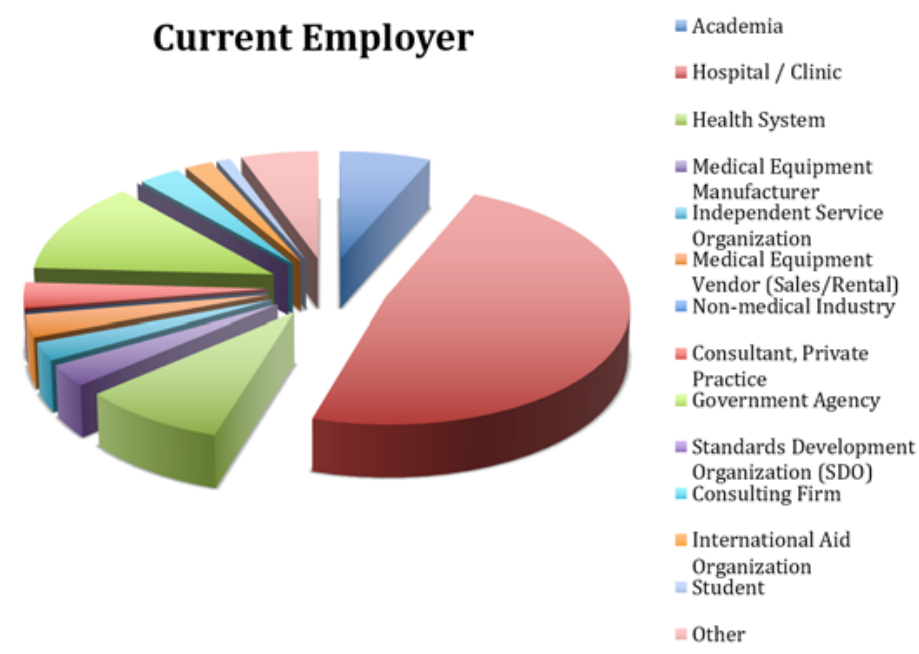


Respondents were also asked how they see themselves as professionals. Nine different names that could define the profession were given (including the options "Others") and the question was presented as: "Which of the following names do you believe best describes your profession?" Around 48\% describe themselves as CEs and $18.1 \%$ as healthcare technology managers.

Asked about the primary nature of his/her present position, the three first positions selected worldwide were Management (45.7\%), Service Delivery (15\%) and Professional Support (14.1\%). However, this question also raised interesting information: Management was the first position in all regions but it was placed second in Oceania (34.15\%) and Service Delivery (41.5\%) was placed first.

Due to the small number of responses from each region, one can argue that such results lack reliability. However, by comparing this information with those obtained in 2015 (the USA and Canada BoK survey,8 with 472 respondents), the picture regarding Technology Management is the same, being in the first position. According to the respondents, there are great variations for other positions depending on the region. This can be seen in Table 2 where Latin American CEs responded (70 responses) that Professional Support is the second position while in the Middle East (21 responses), as Oceania (41 responses). Service Delivery is the second position.

TABLE 2. Final Results of Option Comparison By Category

\begin{tabular}{|c|c|c|c|c|c|c|}
\hline & Africa & Asia & Europe & $\begin{array}{c}\text { Latin } \\
\text { America }\end{array}$ & $\begin{array}{c}\text { Middle } \\
\text { East }\end{array}$ & Oceania \\
\hline Management & $41.67 \%$ & $42.86 \%$ & $50.00 \%$ & $54.29 \%$ & $42.86 \%$ & $34.15 \%$ \\
\hline Research & $0.00 \%$ & $7.14 \%$ & $10.00 \%$ & $4.29 \%$ & $0.00 \%$ & $2.44 \%$ \\
\hline Manufacturing & $0.00 \%$ & $0.00 \%$ & $0.00 \%$ & $1.43 \%$ & $0.00 \%$ & $2.44 \%$ \\
\hline Teaching & $25.00 \%$ & $10.71 \%$ & $0.00 \%$ & $7.14 \%$ & $9.52 \%$ & $2.44 \%$ \\
\hline Consulting & $16.67 \%$ & $14.29 \%$ & $10.00 \%$ & $8.57 \%$ & $0.00 \%$ & $0.00 \%$ \\
\hline $\begin{array}{c}\text { Service } \\
\text { Delivery }\end{array}$ & $8.33 \%$ & $3.57 \%$ & $10.00 \%$ & $4.29 \%$ & $28.57 \%$ & $41.46 \%$ \\
\hline $\begin{array}{c}\text { Professional } \\
\text { Support }\end{array}$ & $8.33 \%$ & $17.86 \%$ & $10.00 \%$ & $15.71 \%$ & $14.29 \%$ & $14.63 \%$ \\
\hline Other & $0.00 \%$ & $3.57 \%$ & $10.00 \%$ & $4.29 \%$ & $4.76 \%$ & $2.44 \%$ \\
\hline
\end{tabular}

One of the most important questions and the one that directly helps to achieve the objective of this project asked respondents to indicate, in a list of background knowledge topics, the level of importance of each one for his/her daily duties and responsibilities. Three levels of importance were presented (Minor, Moderate and High Importance). To present the results, the data processing was already explained in the chapter "Methodology" previously.

Interesting observations can be made by looking at the results presented in Figure 3. Though not with the same level of importance, there are coincident "Knowledge" rating tendencies. All respondent regions rated General Medical/Nursing Equipment above moderate importance. The same happens to the "Knowledge" regarding Computers, Networking and IT. On the other hand, "Knowledge" regarding Telecommunications is below moderate to all regions. The same happens for Chemistry and Implants.

FIGURE 3. Level of importance of background knowledge for clinical engineers to develop their daily work activities.

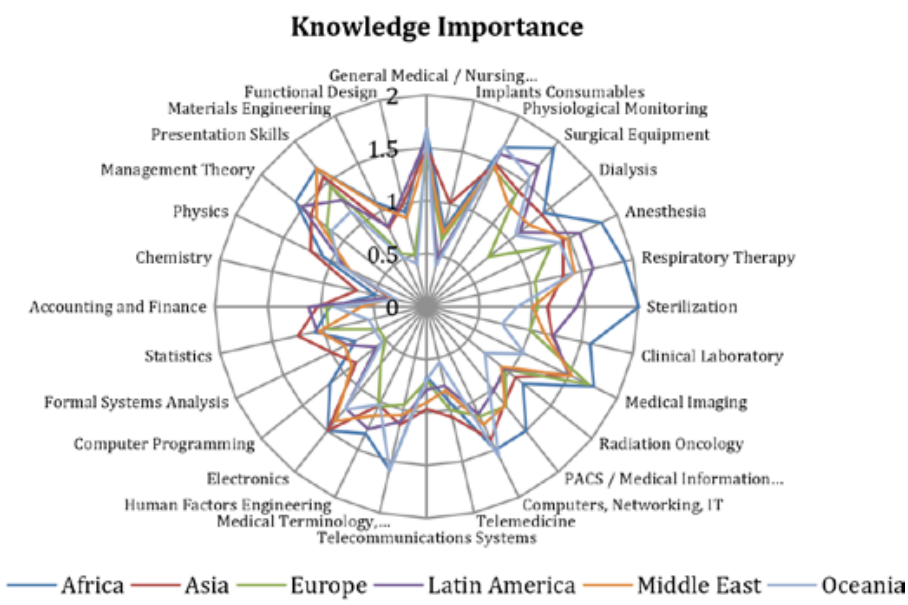

PACS $=$ picture archiving and communication system

Other comments can be made about these results but, the most important one is to see that a primary profile of the Body of Knowledge for CEs all over the world can already be traced, based on the rate tendency.

This survey, as well as the 2005 survey 7 and the one promoted by ACCE9, pointed out that the position of technology manager was the one held by most clinical engineers worldwide. Presented with 20 different 
activities within the scope of this position, they were asked to rate the importance of each activity (High, Moderate, Minor and No) to develop their work. The process used to present this information was already explained in the Methodology chapter.

Again, Figure 4 shows a great number of coincident tendencies regarding the activities. Taking as an example the activity Life Cycle Analysis, all regions rated it between Moderate and High importance. On the other hand, the activity Clinical Trials Management is rated as Minor to Moderate importance within the responsibilities of their work. As mentioned before, despite the low number of respondents, the importance of the activities among the regions follow the same pattern.

FIGURE 4. The importance of activities for clinical engineers within the technology management domain.

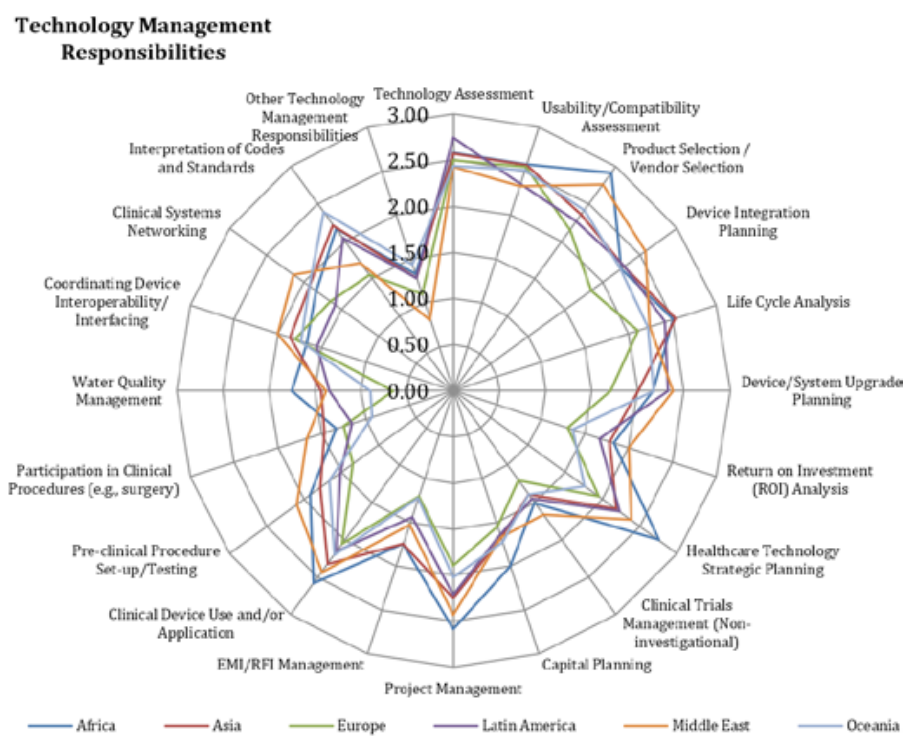

$\mathrm{EMI}$ = electromagnetic interference; RFI = radio frequency interference

Figure 5 presents the percentage of time CEs spent on each work activity during the work. Confirming what was pointed out on Table 2, most of the CEs from the Oceania region spent their time on service delivery (30\%) while CEs from other regions spent between 15\% and 18\% on this activity. Important work profiles can be noticed here; while CEs from the European region spent 11.7\% of their time on Risk Management Safety, this percentage is reduced to $6.7 \%$ by CEs in the Middle Eastern region. Another activity that presents a great difference in the percentage of dedicated time is Education of Others. While in Latin America CEs declared that this activity consumes $13 \%$ of their time, in the European and Oceania regions it drops to $8.5 \%$.

FIGURE 5. Percentage of time spent by clinical engineers on each activity during work.

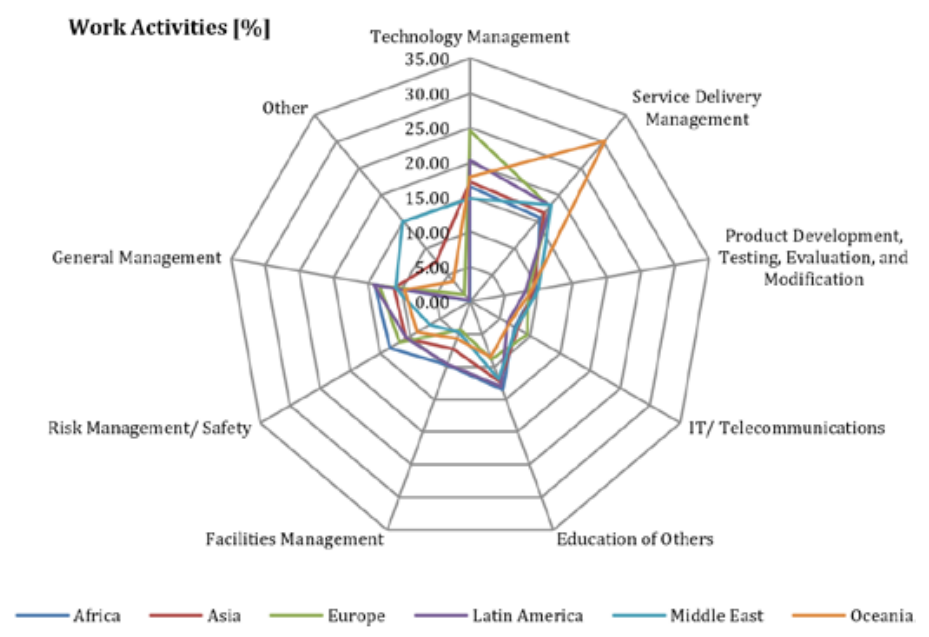

$\mathrm{EMI}=$ electromagnetic interference; RFI = radio frequency interference

The reasons for such percentage differences in some activities may be due to the group of respondents within each region, interpretation of the question (Survey was done only in English) or cultural behavior. One may understand that Education of Others meant a short but formal lecture and others may understand that just the fact of orienting a new technician on repairing medical equipment is part of the time dedicated to education.

The kind of activities the CEs develop for each work activity was also explored, as shown in Figure 5. A total of 18 different activities composing the Risk Management/ Safety work activity was presented to be rated according to its importance to the development of the work.

Figure 6 shows a few differences in the importance of each activity given by the respondents according to the region. While in the Oceanian and European regions the respondents considered Forensic Analysis as low importance, all other regions considered it above moderate. The 
majority of other activities were rated between minor to moderate importance.

FIGURE 6. The importance of each activity within the Work Activity Risk Management/Safety.

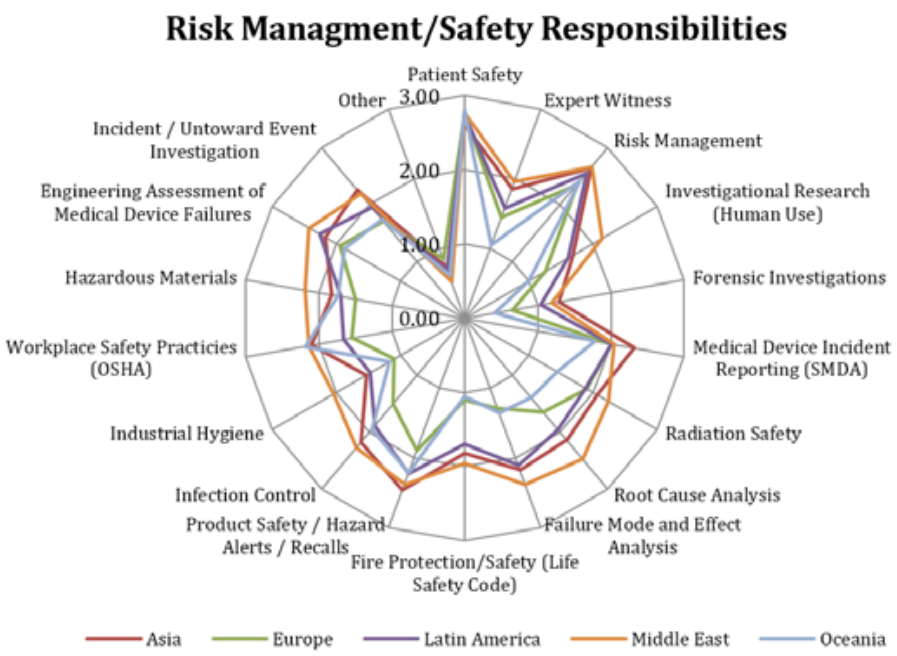

\section{DISCUSSION}

One of the most challenging tasks when designing mainly a worldwide data collection tool in the form of a survey is to develop a question that has the exact meaning to all respondents. Due to language and culture differences as well as different academic systems and job titles, people tend to respond according to the regional characteristics, which cause some distortions in the analysis of the results. Some of the data obtained can be corrected by a simple translation to English while others would be necessary to have a deeper understanding of the country's academic system.

Adaptations from the ACCE survey were necessary to meet the objectives of this survey. It was not only to identify the CE body of practice and CE profiles worldwide but also to use the identified the body of practice to understand the body of knowledge required by CEs to successfully develop such activities. It is expected that in the near future this set of knowledge would help to develop a scope of academic subjects necessary for graduating students to understand to optimally practice such activities.
The structure of the survey allows a more detailed analysis of the data obtained. It is possible, according to the answers and number of responses, to have the profile not only of each respondent, but also the CE model practiced by the country, and the health unit he/she is working at.

No doubt that a higher number of responses from clinical engineers and other countries would make the information more accurate. However, it is already possible to devise a core of activities practiced by CEs all over the world. Regarding the needed knowledge for better developing their work, the results showed that though its importance varies according to the CE model practiced in the country/region, there is also a set of knowledge that is commonly needed worldwide.

There is a need to periodically update the information obtained in this survey due to the dynamic characteristic of the CE profession and the changing dependence of healthcare services on technology. For almost every new technology and procedure to be used in the health area there is an anticipated and required a new set of knowledge for the practicing clinical engineers throughout the technology life cycle stages from innovation to disposal and replacement.

\section{CONCLUSION}

We hypothesized that a common body of knowledge and body of practice for CE would emerge from the analysis of a worldwide survey.

Despite some differences between regions, some patterns of perceived relevance of different fields of knowledge and activity responsibilities within the areas are visible.

This suggests that CE does have something in common around the world but more responses are necessary to define a solid worldwide Body of Knowledge and Body of Practice for the Profession.

\section{ACKNOWLEDGEMENTS}

The authors wish to thank Eng. Frank Painter for authorizing the adaptation and use of an earlier version of the survey he developed. 


\section{REFERENCES}

1. US Department of Labor - Bureau of Labor Statistics. Occupational Employment and Wages, May 2017. Available at: https://www.bls.gov/oes/2017/may/ oes172031.htm

2. Judd, T., \& David, Y. (2018). Making a Difference Global Health Technology Success Stories: Overview of over 400 submissions from 125 Countries. Global Clinical Engineering Journal, 1(1), 24-49. https://doi. org/10.31354/globalce.v1i1.4

3. Frize M. Results of an international survey of clinical engineering departments - Part 2. Med Biol Eng Computing 1990;28:160. https://doi.org/10.1007/ BF02441772

4. Nagel J. BIOMEDEA. In: Jarm T, Kramar P, Zupanic A. (eds) 11th Mediterranean Conference on Medical and Biomedical Engineering and Computing 2007. IFMBE Proceedings, vol 16. Springer, Berlin, Heidelberg; 2007. Available at: https://link.springer.com/ chapter/10.1007/978-3-540-73044-6_289

5. Nagel J. BIOMEDEA Invitation. Stuttgart: Institut für Biomedizinische Technik; 2005. Available at: http:// www.vvb-bmt.nl/EmailAlerts/attach/20050722-01.pdf
6. Wear J. Certification/Registration of Clinical Engineering Practitioners. CED/IFMBE 2017. Available at: http:// cedglobal.org/wp-content/uploads/2018/06/CEDCertification-White-Paper-with-Annexes.pdf

7. Calil S. J., Nascimento L. N., Painter F. R. (2007) Findings of the Worldwide Clinical Engineering Survey conducted by the Clinical Engineering Division of the International Federation for Medical and Biological Engineering. IFMBE Proc. vol. 16. 11th Mediterranean Conference on Medical and Biomedical Engineering and Computing 2007, pp 1085-1088. https://doi. org/10.1007/978-3-540-73044-6_280

8. Calil. S. J., Oliveira R. R. V., Nascimento L. N. (2009) "Findings of the Latin America Clinical Engineering Survey Conducted by the Clinical Engineering Division of International Federation for Medical and Biological Engineering." World Congress on Medical Physics and Biomedical Engineering. September 7-12. 2009. Munich. Germany. Springer. Berlin. Heidelberg.

9. Suhan A. 2015 American College of Clinical Engineering Body of Knowledge Survey Results. J Clin Engineer 2017;42(3). 